\title{
DNA methylation patterns of candidate genes regulated by thymine DNA glycosylase in patients with TP53 germline mutations
}

\author{
F.P. Fortes ${ }^{1}$, H. Kuasne ${ }^{2,3}$, F.A. Marchi ${ }^{2,4}$, P.M. Miranda ${ }^{2}$, S.R. Rogatto ${ }^{2,3}$ and M.I. Achatz ${ }^{1,5}$ \\ ${ }^{1}$ CIPE, Laboratório de Oncogenética Molecular, A.C. Camargo Cancer Center, São Paulo, SP, Brasil \\ ${ }^{2}$ CIPE, Laboratório NeoGene, A.C. Camargo Cancer Center, São Paulo, SP, Brasil \\ ${ }^{3}$ Departamento de Urologia, Faculdade de Medicina, Universidade Estadual Paulista, Botucatu, SP, Brasil \\ ${ }^{4}$ Programa Inter-Institucional em Bioinformática, Instituto de Matemática e Estatística, \\ Universidade São Paulo, São Paulo, SP, Brasil \\ ${ }^{5}$ Departamento de Oncogenética, A.C. Camargo Cancer Center, São Paulo, SP, Brasil
}

\begin{abstract}
Li-Fraumeni syndrome (LFS) is a rare, autosomal dominant, hereditary cancer predisposition disorder. In Brazil, the p.R337H TP53 founder mutation causes the variant form of LFS, Li-Fraumeni-like syndrome. The occurrence of cancer and age of disease onset are known to vary, even in patients carrying the same mutation, and several mechanisms such as genetic and epigenetic alterations may be involved in this variability. However, the extent of involvement of such events has not been clarified. It is well established that p53 regulates several pathways, including the thymine DNA glycosylase (TDG) pathway, which regulates the DNA methylation of several genes. This study aimed to identify the DNA methylation pattern of genes potentially related to the TDG pathway (CDKN2A, FOXA1, HOXD8, OCT4, SOX2, and SOX17) in 30 patients with germline TP53 mutations, 10 patients with wild-type TP53, and 10 healthy individuals. We also evaluated TDG expression in patients with adrenocortical tumors (ADR) with and without the p.R337H TP53 mutation. Gene methylation patterns of peripheral blood DNA samples assessed by pyrosequencing revealed no significant differences between the three groups. However, increased $T D G$ expression was observed by quantitative reverse transcription PCR in p.R337H carriers with ADR. Considering the rarity of this phenotype and the relevance of these findings, further studies using a larger sample set are necessary to confirm our results.
\end{abstract}

Key words: Li-Fraumeni syndrome; TP53 gene; TDG; Methylation

\section{Introduction}

Li-Fraumeni syndrome (LFS, OMIM \#151623) is an autosomal dominant disorder characterized by an inherited predisposition to cancer and the development of multiple primary tumors at an early age. The cancers most frequently associated with LFS are breast cancer, adrenocortical carcinoma (ADR), soft tissue sarcoma, osteosarcoma, and central nervous system tumors (1-4).

The main molecular mechanism underlying LFS is germline mutations in TP53 (5), which predominantly occur in the central DNA-binding domain (6). In Southern Brazil, a variant form of LFS, Li-Fraumeni-like syndrome (LFL), occurs as a result of a founder mutation in exon 10 of TP53, replacing an arginine with histidine at codon 337 (p.R337H), which falls within the oligomerization domain (7). The p.R337H mutation alters the functional properties of the p53 protein at elevated intracellular pH values (above 7.0 ) and/or temperatures above $36.5^{\circ} \mathrm{C}$ $(7,8)$.

Recent reports have indicated that TP53 mutations indirectly alter the levels of several transcripts (9), including the specialized base excision repair enzyme thymine-DNA glycosylase (TDG) (10). The primary role of TDG is to correct guanine:thymine and guanine:uracil DNA mismatches that result from the spontaneous deamination of 5-methyl cytosine and cytosine at CpG sites. These mutations can result in the loss of $\mathrm{CpG}$ dinucleotides, potentially affecting gene regulation $(11,12)$. Léger et al. (13) demonstrated that the introduction of a P65A point mutation in TDG led to a significant loss of TDG/CREB-binding protein/retinoic acid receptor $\alpha$ ternary complex stability, resulting in the deregulation of networks associated with DNA replication, recombination,

Correspondence: M.I. Achatz: <miachatz@cipe.accamargo.org.br>.

Received May 1, 2014. Accepted February 5, 2015. First published online April 28, 2015. 
and repair. TDG is also involved in the physiological control of promoter demethylation of several genes that are involved in embryogenesis and development (14-16), and it acts as a positive Wnt pathway regulator in patients with colorectal cancer (17).

Cells lacking TDG activity exhibit two major alterations: a decreased capacity for base excision repair, leading to increased sensitivity to mutagenic damage and the accumulation of mutations, and an impaired ability to maintain wild-type promoter region methylation patterns, resulting in inappropriate gene expression. Both TDG and ten-eleven-translocation (TET) protein mediate the demethylation and reactivation of micro (mi)RNAs that are critical for the mesenchymal-to-epithelial transition (18).

Alterations in the normal methylation patterns of TDGregulated genes may be one mechanism underlying the occurrence of early age tumors in patients with germline TP53 mutations. Indeed, the presence of high methylation levels in the promoter regions of certain genes has been considered to be a marker for several tumors (19-21). Epigenetic alterations, particularly DNA methylation, are a plausible molecular mechanism that may contribute to the diversity of tumors described in LFS/LFL patients.

p53 is known to alter TDG expression, which then modifies the methylation of genes related to embryogenesis and development. Our objective for the present study was therefore to evaluate the methylation patterns of six genes that are likely to be dependent on TDG activity, aiming to verify its relevance in patients carrying the p.R337H mutation. This group of genes produces transcripts that are related to pluripotency (OCT4 and SOX2), a transcription factor involved in morphogenesis and a homeobox family member (HOXD8), a regulator of development (SOX17), a replicative senescence controller $(C D K N 2 A)$, and a transcription factor related to embryonic development (FOXA1). We also used a retrotransposon sequence with constitutive, stable methylation (ALUyB8) as a DNA methylation control.

\section{Material and Methods}

Fifty individuals recruited from the Oncogenetics Department of the A.C. Camargo Cancer Center (São Paulo, SP, Brazil) were selected for methylation analysis and divided into five groups: 1) 10 patient $p . R 337 \mathrm{H}$ carriers that had developed cancer, 2) 10 patient p.R337H carriers without cancer, 3) 10 individuals with cancer and carrying germline TP53 mutations other than p.R337H, 4) 10 individuals with wild-type TP53 and relatives who are carriers of germline TP53 mutations, and 5) 10 healthy individuals with no personal or family history of cancer (Supplementary Table S1). All methylation assays were performed on DNA extracted from peripheral blood samples. Adrenocortical carcinomas from two patients with the p.R337H mutation and six patients without it were selected for gene expression analysis (Supplementary
Table S2). The Oncogenetics Department of the A.C. Camargo Cancer Center followed up all patients. The Institutional Review Board approved this study (\#1669/12).

\section{DNA and RNA extraction}

DNA was extracted from peripheral blood samples using a Gentra Puregene Blood kit (Qiagen, USA) according to the manufacturer's instructions, quantified using a NanoDrop ND-1000 Spectrophotometer v.3.0.1 (Thermo Scientific, USA) and stored at $-20^{\circ} \mathrm{C}$.

Total RNA was obtained from adrenocortical carcinomas using an RNeasy kit (Qiagen) according to the manufacturer's recommendations. The quantity and quality of isolated RNAs were assessed using a NanoDrop ND-1000 Spectrophotometer v.3.0.1 (Thermo Scientific), and an Agilent 2100 Bioanalyzer (Agilent Technologies, USA) combined with an RNA 6000 NanoLabChip kit 2100 (Agilent Technologies), respectively. DNAs and RNAs were extracted from the samples at the A.C. Camargo Cancer Biobank (Brazil).

\section{Investigation of the germline TP53 p.R337H mutation}

Exon 10 of TP53 was amplified using primer sequences 5'-CAA CTT TTG TAA GAA CCA TC-3' and 5'-GGA TGA GAA TGG AAT CCT AT-3' (22). Briefly, the amplification consisted of 35 cycles of denaturation at $94^{\circ} \mathrm{C}$, annealing at $57^{\circ} \mathrm{C}$, and extension at $68^{\circ} \mathrm{C}$. The PCR products were digested with $1 \mathrm{U} / \mu \mathrm{L}$ Hhal (Fermentas Inc., USA) for $16 \mathrm{~h}$ at $37^{\circ} \mathrm{C}$, run on a $2 \%$ agarose gel $(1 \times$ Tris-borate-EDTA buffer), and the following digestion patterns observed: 168 and 92 bp bands indicating normal homozygous cells, 260 , 168, and $92 \mathrm{bp}$ bands indicating p.R337H heterozygous cells, and a single $260 \mathrm{bp}$ band indicating p.R337H homozygous cells (23).

In addition to the p.R337H mutation, we also examined TP53 exons 2-11, including the flanking intronic regions containing splice sites using protocols from the International Agency for Research on Cancer (http://p53. iarc.fr/Download/TP53_DirectSequencing_IARC.pdf). Sanger sequencing was conducted as described by Coulson (24). PCR amplification used a GeneAmp PCR System 9700 (Applied Biosystems, USA) and sequencing was performed using an ABI Prism Model 3130xI (Applied Biosystems) automatic sequencer. The resulting sequences were comparatively analyzed using a reference sequence (RefSeq NM_000546.4) and the CLC Main Workbench 5.0 .2 software (Denmark). This analysis included all exons and exon-intron junctions.

\section{Pyrosequencing investigation of methylated CpG islands}

The presence of methylated $\mathrm{CpG}$ islands was examined in six genes: FOXA1, OCT4, SOX17, CDKN2A, HOXD8, and SOX2. A total of $500 \mathrm{ng}$ of DNA from each sample was treated with bisulfite using an EZ DNA Methylation Kit-Gold kit (Zymo Research, USA). FOXA1, 
OCT4, and SOX17 amplification primers are described in Table 1. Standardized Qiagen tests were used for CDKN2A, HOXD8, and SOX2 (Table 1).

PCR amplifications were performed in $50-\mu \mathrm{L}$ volumes containing $1-\mu \mathrm{L}$ converted DNA (25 ng), $10 \times$ buffer, $15 \mathrm{mM} \mathrm{MgCl}$, $10 \mathrm{mM}$ of each dinucleotide, $10 \mathrm{mM}$ of each primer (one labeled with biotin at the $5^{\prime}$ end), and $1 \mathrm{U}$ HotStartTaq DNA polymerase (Qiagen). Pyrosequencing reactions were performed using Pyromark Gold Q96 reagents (Qiagen) according to the manufacturer's recommendations. Significant differences between groups were determined using the Kruskal Wallis test.

\section{cDNA synthesis and quantitative reverse transcription PCR analysis}

To assess changes in TDG expression, RNA samples from eight adrenocortical carcinomas were used for CDNA synthesis as previously described (25). Quantitative reverse transcription (qRT)-PCR was performed using Power SYBR ${ }^{\circledR}$ Green fluorescent dye (Applied Biosystems) in an ABI Prism 7500 Sequence Detection
System. All sample values were normalized by dividing the values obtained for the gene of interest (TDG) with those for the reference genes (HPRT and GAPDH). The primers for transcript amplification were designed using the Primer Blast program (http://www.ncbi.nlm.nih.gov/ tools/primer-blast/) (Supplementary Table S3). Target gene quantification was performed using $\mathrm{Ct}$ values and the $2^{\Delta \Delta C t}$ formula (26). Expression values were compared with a control sample that consisted of a commercial RNA pool from normal adrenal tissue (Clontech, USA).

\section{Results}

We identified 20 TP53 p.R337H carrier patients, 10 patients with other TP53 mutations, and 20 patients with wild-type TP53. Clinical and biological characteristics of the LFS/LFL patients, as well as the sequencing results for each case, are described in Supplementary Table S1.

The methylation patterns of CDKN2A, FOXA1, HOXD8, OCT4, SOX2, and SOX17 were evaluated by pyrosequencing. As shown in Figure 1, no significant

Table 1. Primer sequences and the properties of the genes assessed by pyrosequencing.

\begin{tabular}{|c|c|c|c|c|c|}
\hline Gene & Assay & Primers sequence & $\begin{array}{l}\text { Amplicon } \\
\text { (bp) }\end{array}$ & $\begin{array}{l}\mathrm{CpG}^{*} \\
\text { (n) }\end{array}$ & $\begin{array}{l}\text { Annealing } \\
\text { temperature }\end{array}$ \\
\hline \multirow[t]{4}{*}{ FOXA1 } & - & F:5'TTTAGTTGTGGGGAGGATGG & 191 & $\ldots$ & $55^{\circ} \mathrm{C}$ \\
\hline & & R:5'[B] CACCCTACAATCCTCACTAC & & & \\
\hline & & Seq:5'TGTTTGTGTTTAAGA & & & \\
\hline & & Py:5'CGGGTTTGCGATAGTTTGGGGCGGTTIAGGTCGCG & & & \\
\hline \multirow[t]{4}{*}{ OCT4 } & - & F:5'TAGGAGGGTTTTGGAAGTTTAG3' & 174 & $\ldots$ & $55^{\circ} \mathrm{C}$ \\
\hline & & R:5'[B]AAACCCTCATTTCACCAAAC3' & & & \\
\hline & & Seq:5'TTATTATTTGGAGGGGG3' & & & \\
\hline & & $\begin{array}{c}\text { Py:5'CGCGATTTCGGTTTATCG } \\
\text { TAATTTATATTTTTTAGGTTTAAGCGA3' }\end{array}$ & & & \\
\hline \multirow[t]{4}{*}{ SOX17 } & - & F:5'GTTGTTTTATTTGGGAGGTG3' & 369 & $\ldots$ & $55^{\circ} \mathrm{C}$ \\
\hline & & R:5'[B] ACCCCTAAATAACCAAACAAA3' & & & \\
\hline & & Seq:5'GATTTGTTTTTTAG3' & & & \\
\hline & & Py:5'CGTTAATTCGGTATTGTTTAGGTGTTTACG3' & & & \\
\hline SOX2 & $\begin{array}{c}\text { Hs_SOX2_01_PM } \\
\text { PyroMark CpG Assay } \\
\text { (200) (PM00016856) }\end{array}$ & 5'CCCCGGCGCCGAGGTGCCGGACCGCCGCCCCCAGC3' & 205 & $\ldots$ & $56^{\circ} \mathrm{C}$ \\
\hline HOXD8 & $\begin{array}{l}\text { Hs_HOXD8_02_PM } \\
\text { PyroMark CpG Assay } \\
\text { (200) (PM00012026) }\end{array}$ & 5'CGTTCCCTGGGCTGCACCCGCGTGTCCAGAGCTGC3' & 155 & 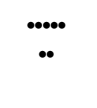 & $56^{\circ} \mathrm{C}$ \\
\hline$C D K N 2 A$ & $\begin{array}{c}\text { Hs_CDKN2A_02_PM } \\
\text { PyroMark Cp } \bar{G} \text { Assay } \\
\text { (200) (PM00039907) }\end{array}$ & 5'CGCCGTGAGCGAGTGCTCGGAGGAGGTGCTATTAA3' & 249 & 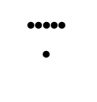 & $56^{\circ} \mathrm{C}$ \\
\hline
\end{tabular}

F: primer forward; R: primer reverse; Seq; sequencing region; Py: reference sequence (CG in bold at the CpG sites were analyzed by pyrosequencing); bp base pairs; $\bullet$ number of $\mathrm{CpG}$ analyzed by gene; * number of $\mathrm{CpG}$ sites evaluated. 
differences were observed when all tested patient groups were evaluated. The methylation levels of $C D K N 2 A$, SOX2, SOX17, and HOXD8 were below $5 \%$ in all five groups. Additionally, FOXA1 showed methylation levels below $15 \%$ in all groups. OCT4 and ALUyb8 methylation levels were approximately $80 \%$.

In the adrenocortical carcinoma samples, two of the eight tumors possessed the p.R337H mutation (Supplementary Table S2). RT-qPCR also revealed higher TDG expression in both p. $\mathrm{R} 337 \mathrm{H}$-positive cases; however, this finding could not be tested for statistical significance because of the small sample size.

\section{Discussion}

LFS patients have a $90 \%$ risk of developing cancer during their lifetime (27). According to Chompret criteria, germline mutations in TP53 are found in 70\% of LFS cases (28) and in $29 \%$ of LFL families (29). The TP53 p.R337H founder mutation was reported to be associated with Brazilian families with LFL in 2007 (2), and has an estimated population frequency of $0.3 \%$ in Southern and Southeastern regions of Brazil, where the incidence of adrenocortical carcinoma is 10 - to 15 -fold greater than in other countries $(30,31)$.

Recently, da Costa et al. (10) reported that TDG expression is directly regulated by wild-type p53 protein, suggesting that the loss of p53 function may affect TDGmediated processes. A limited number of studies have assessed TDG expression levels in tumors. Nettersheim et al. (32) reported high levels of TDG and TET transcripts in germ cell-derived tumors, while Peng et al. (33) reported that TDG hypermethylation and the consequent reduction of transcript expression led to an impairment of repair in multiple myeloma cell lines. Similarly, Yatsuoka et al. (34) observed decreased TDG expression in 21 pancreatic cancer cell lines. Interestingly, TDG expression levels appear to be epigenetically regulated by DNA methyltransferases, especially DNMT3L (35), and the miRNA-29 family (36).

In addition to its involvement in DNA damage repair, TDG has been shown to be involved in epigenetic regulation, protecting $\mathrm{CpG}$ islands from hypermethylation through interactions with DNA methyltransferases and histone acetyltransferases. Moreover, TDG glycosylase activity plays an active role in 5-methylcytosine removal and thus leads to gene activation through demethylation $(10,37,38)$. TDG is also very active during development (15), and epigenetically regulates several genes associated with development and cell determination such as the homeobox family genes and other transcription factors (15).

The present study evaluated the methylation patterns of CDKN2A, FOXA1, HOXD8, OCT4, SOX2, and SOX17 in peripheral blood samples from patients with germline
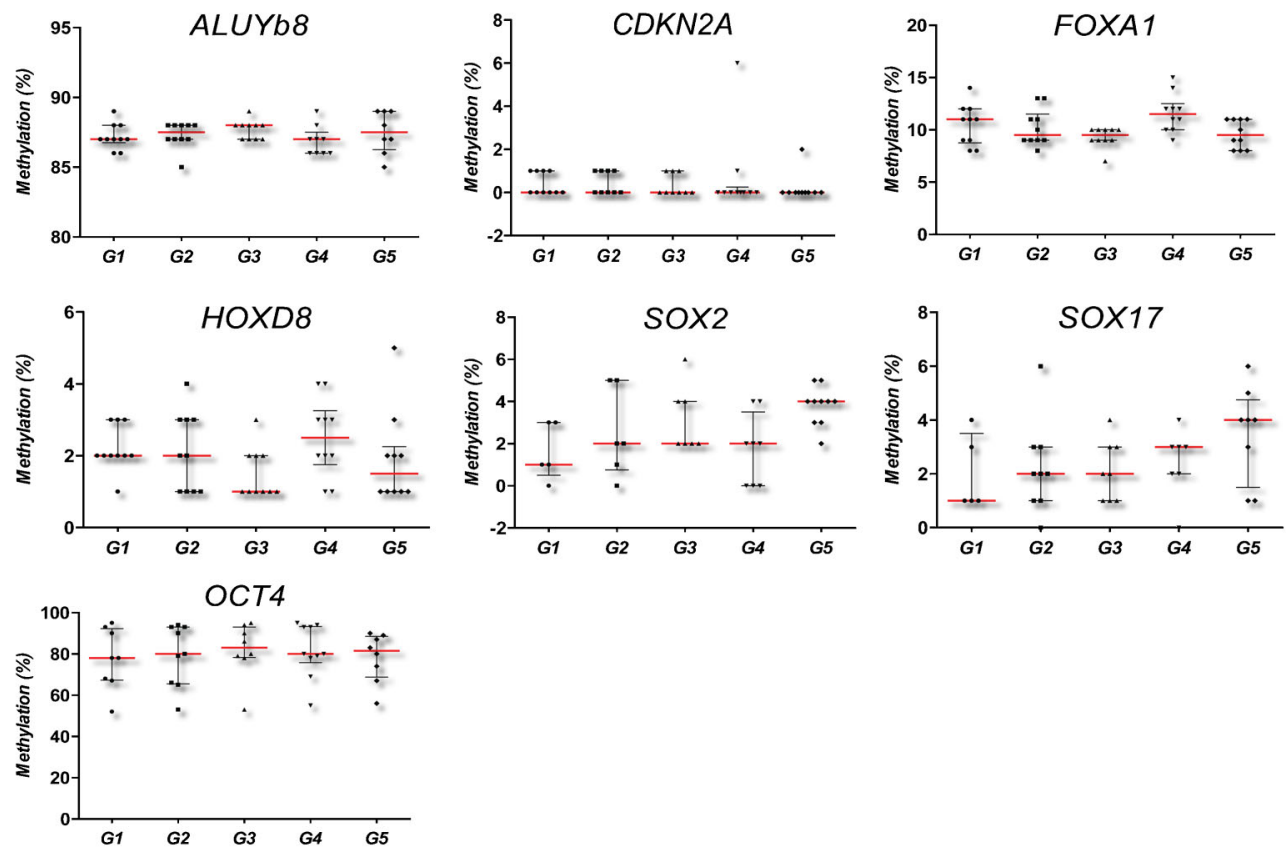

Figure 1. Dot plots representing the methylation levels of CDKN2A, FOXA1, HOXD8, SOX2, SOX17 and OCT4 candidate genes, as well as of the ALUYb8 control region, in groups G1 through G5. Groups: G1: 10 patient p.R337H carriers that had developed cancer; G2: 10 patient p.R337H carriers without cancer; G3: 10 individuals with cancer and carrying germline TP53 mutations other than p.R337H; G4: 10 individuals with wild-type TP53 and relatives who are carriers of germline TP53 mutations; G5: 10 healthy individuals with no personal or family history of cancer. There were no significant differences ( $P>0.05$, Kruskal-Wallis test). 
TP53 mutations and healthy individuals. The six genes selected are related to development and embryogenesis and are potentially regulated by TDG.

Methylation profiles may differ in various tissues within a single individual (39). The assessment of methylation status in both tumor and peripheral blood samples therefore has the potential to reveal differences that could help us better understand the tumor variability and penetrance observed in LFS TP53 germline mutation carriers. Methylation pattern analysis using peripheral blood samples is also an effective, non-invasive alternative to investigating the tumor spectrum variability within the syndrome. Our initial hypothesis was that epigenetic alterations would be observed in blood samples from LFS/LFL patients or that altered methylation patterns could indicate indirect alterations to TDG expression.

LFS/LFL patients display a variety of tumor types over a wide age spectrum, and it has been observed that even if patients carry the same mutation, they do not always exhibit the same phenotype (40). Alterations to the methylation patterns of genes potentially regulated by TDG could act as risk modifiers, and could explain the differences in the ages of tumor onset and tumor subtypes described in this syndrome; however, we were unable to confirm the differences in the methylation patterns of the tested genes and samples (Figure 1). Nevertheless, our LFS/LFL patient cohort is one of the largest described with germline TP53 mutations, even though we had a restricted number of patients who fulfilled the inclusion criteria. None of the genes evaluated in LFS/LFL patients showed hypermethylation compared with controls, so they

\section{References}

1. Malkin D, Li FP, Strong LC, Fraumeni JF Jr, Nelson CE, $\mathrm{Kim} \mathrm{DH}$, et al. Germ line p53 mutations in a familial syndrome of breast cancer, sarcomas, and other neoplasms. Science 1990; 250: 1233-1238, doi: 10.1126/science. 1978757.

2. Achatz MI, Olivier M, Le Calvez F, Martel-Planche G, Lopes $A$, Rossi BM, et al. The TP53 mutation, $\mathrm{R} 337 \mathrm{H}$, is associated with Li-Fraumeni and Li-Fraumeni-like syndromes in Brazilian families. Cancer Lett 2007; 245: 96102, doi: 10.1016/j.canlet.2005.12.039.

3. Marcel V, Dichtel-Danjoy ML, Sagne C, Hafsi H, Ma D, Ortiz-Cuaran S, et al. Biological functions of p53 isoforms through evolution: lessons from animal and cellular models. Cell Death Differ 2011; 18: 1815-1824, doi: 10.1038/ cdd.2011.120.

4. Palmero El, Achatz MI, Ashton-Prolla P, Olivier M, Hainaut $P$. Tumor protein 53 mutations and inherited cancer: beyond Li-Fraumeni syndrome. Curr Opin Oncol 2010; 22: 64-69, doi: 10.1097/CCO.0b013e328333bf00.

5. Frebourg T, Malkin D, Friend S. Cancer risks from germ line tumor suppressor gene mutations. Princess Takamatsu Symp 1991; 22: 61-70. cannot be used as markers for the assessment of LFS/ LFL phenotypes. The use of more robust platforms (e.g., large scale analysis) or next-generation sequencing to assess epigenetic alterations is likely to be more effective in finding TDG-regulated genes or other markers to evaluate such phenotypic differences. It is also worth noting that methylation is labile and thus may be influenced by several factors such as life habits and age. Although increased TDG expression was observed in two adrenocortical carcinomas from patients who were positive for the $\mathrm{p} . \mathrm{R} 337 \mathrm{H}$ mutation, it was not possible to infer the relationship between the $\mathrm{p} . \mathrm{R} 337 \mathrm{H}$ mutation and TDG levels because of the small number of cases. A larger cohort of patients with matched controls is therefore needed to better assess TDG as a clinical marker for tumor occurrence in LFS families; however, this disease is a rare syndrome and the recruitment of a large number of patients remains a challenge.

\section{Supplementary Material}

Click here to view [pdf]

\section{Acknowledgments}

The authors would like to thank the A.C. Camargo Biobank and Dr. Antônio Hugo J.F.M. Campos (São Paulo, Brazil). This research was supported by the National Institute of Science and Technology in Oncogenomics (INCITO-\#573589/2008-9) and CNPq (\#830068/2001-5).

6. Olivier M, Goldgar DE, Sodha N, Ohgaki $H$, Kleihues $P$, Hainaut $\mathrm{P}$, et al. Li-Fraumeni and related syndromes: correlation between tumor type, family structure, and TP53 genotype. Cancer Res 2003; 63: 6643-6650.

7. DiGiammarino EL, Lee AS, Cadwell C, Zhang W, Bothner B, Ribeiro RC, et al. A novel mechanism of tumorigenesis involving $\mathrm{pH}$-dependent destabilization of a mutant p53 tetramer. Nat Struct Biol 2002; 9: 12-16, doi: 10.1038/nsb730.

8. Hainaut $P$. Tumor-specific mutations in p53: the acid test. Nat Med 2002; 8: 21-23, doi: 10.1038/nm0102-21.

9. Parikh N, Hilsenbeck S, Creighton CJ, Dayaram T, Shuck R, Shinbrot E, et al. Effects of TP53 mutational status on gene expression patterns across 10 human cancer types. J Pathol 2014; 232: 522-533, doi: 10.1002/path.4321.

10. da Costa NM, Hautefeuille A, Cros MP, Melendez ME, Waters $T$, Swann $P$, et al. Transcriptional regulation of thymine DNA glycosylase (TDG) by the tumor suppressor protein p53. Cell Cycle 2012; 11: 4570-4578, doi: 10.4161/ cc.22843.

11. Ehrlich M, Zhang XY, Inamdar NM. Spontaneous deamination of cytosine and 5-methylcytosine residues in DNA and replacement of 5-methylcytosine residues with cytosine 
residues. Mutat Res 1990; 238: 277-286, doi: 10.1016/ 0165-1110(90)90019-8.

12. Krokan HE, Drablos F, Slupphaug G. Uracil in DNA occurrence, consequences and repair. Oncogene 2002; 21: 8935-8948, doi: 10.1038/sj.onc.1205996.

13. Léger $\mathrm{H}$, Smet-Nocca $\mathrm{C}$, Attmane-Elakeb A, MorleyFletcher S, Benecke AG, Eilebrecht S. A TDG/CBP/ RARalpha ternary complex mediates the retinoic aciddependent expression of DNA methylation-sensitive genes. Genomics Proteomics Bioinformatics 2014; 12: 8-18, doi: 10.1016/j.gpb.2013.11.001.

14. Maiti A, Drohat AC. Thymine DNA glycosylase can rapidly excise 5-formylcytosine and 5-carboxylcytosine: potential implications for active demethylation of CpG sites. J Biol Chem 2011; 286: 35334-35338, doi: 10.1074/jbc. C111.284620

15. Cortazar D, Kunz C, Selfridge J, Lettieri T, Saito $Y$, MacDougall E, et al. Embryonic lethal phenotype reveals a function of TDG in maintaining epigenetic stability. Nature 2011; 470: 419-423, doi: 10.1038/nature09672.

16. Cortellino S, Xu J, Sannai M, Moore R, Caretti E, Cigliano A, et al. Thymine DNA glycosylase is essential for active DNA demethylation by linked deamination-base excision repair. Cell 2011; 146: 67-79, doi: 10.1016/j.cell.2011.06.020.

17. $\mathrm{Xu} \mathrm{X,} \mathrm{Yu} \mathrm{T,} \mathrm{Shi} \mathrm{J,} \mathrm{Chen} \mathrm{X,} \mathrm{Zhang} \mathrm{W,} \mathrm{Lin} \mathrm{T,} \mathrm{et} \mathrm{al.} \mathrm{Thymine}$ DNA glycosylase is a positive regulator of Wnt signaling in colorectal cancer. J Biol Chem 2014; 289: 8881-8890, doi: 10.1074/jbc.M113.538835.

18. Hu X, Zhang L, Mao SQ, Li Z, Chen J, Zhang RR, et al. Tet and TDG mediate DNA demethylation essential for mesenchymal-to-epithelial transition in somatic cell reprogramming. Cell Stem Cell 2014; 14: 512-522, doi: 10.1016/ j.stem.2014.01.001.

19. Jones PA, Takai D. The role of DNA methylation in mammalian epigenetics. Science 2001; 293: 1068-1070, doi: 10.1126/science.1063852.

20. Sanchez-Cespedes M, Esteller M, Wu L, Nawroz-Danish H, Yoo GH, Koch WM, et al. Gene promoter hypermethylation in tumors and serum of head and neck cancer patients. Cancer Res 2000; 60: 892-895.

21. Esteller M, Corn PG, Baylin SB, Herman JG. A gene hypermethylation profile of human cancer. Cancer Res 2001; 61: 3225-3229.

22. Petitjean A, Mathe E, Kato S, Ishioka C, Tavtigian SV, Hainaut $P$, et al. Impact of mutant p53 functional properties on TP53 mutation patterns and tumor phenotype: lessons from recent developments in the IARC TP53 database. Hum Mutat 2007; 28: 622-629, doi: 10.1002/humu.20495.

23. Sambrook J, Russell DW. Molecular cloning: a laboratory manual. Vol. 2. Gel eletroforese. 3rd edn. New York: Cold Spring Harbor; 2001.

24. Sanger F, Coulson AR. A rapid method for determining sequences in DNA by primed synthesis with DNA polymerase. J Mol Biol 1975; 94: 441-448, doi: 10.1016/00222836(75)90213-2.

25. Rosa FE, Caldeira JR, Felipes J, Bertonha FB, Quevedo $F C$, Domingues MA, et al. Evaluation of estrogen receptor alpha and beta and progesterone receptor expression and correlation with clinicopathologic factors and proliferative marker Ki-67 in breast cancers. Hum Pathol 2008; 39: 720 730, doi: 10.1016/j.humpath.2007.09.019.
26. Livak KJ, Schmittgen TD. Analysis of relative gene expression data using real-time quantitative PCR and the 2(-Delta Delta C(T)) Method. Methods 2001; 25: 402-408, doi: 10.1006/meth.2001.1262.

27. Nagy R, Sweet K, Eng C. Highly penetrant hereditary cancer syndromes. Oncogene 2004; 23: 6445-6470, doi: 10.1038/sj.onc. 1207714.

28. Varley JM, McGown G, Thorncroft M, James LA, Margison GP, Forster G, et al. Are there low-penetrance TP53 Alleles? evidence from childhood adrenocortical tumors. Am J Hum Genet 1999; 65: 995-1006, doi: 10.1086/302575.

29. Bougeard G, Sesboue R, Baert-Desurmont S, Vasseur S, Martin $\mathrm{C}$, Tinat $\mathrm{J}$, et al. Molecular basis of the Li-Fraumeni syndrome: an update from the French LFS families. $J$ Med Genet 2008; 45: 535-538, doi: 10.1136/jmg.2008.057570.

30. Sandrini R, Ribeiro RC, DeLacerda L. Childhood adrenocortical tumors. J Clin Endocrinol Metab 1997; 82: 20272031.

31. Custodio G, Parise GA, Kiesel FN, Komechen H, Sabbaga $\mathrm{CC}$, Rosati R, et al. Impact of neonatal screening and surveillance for the TP53 R337H mutation on early detection of childhood adrenocortical tumors. $J$ Clin Oncol 2013; 31: 2619-2626, doi: 10.1200/JCO.2012.46.3711.

32. Nettersheim D, Heukamp LC, Fronhoffs F, Grewe MJ, Haas $\mathrm{N}$, Waha A, et al. Analysis of TET expression/activity and $5 \mathrm{mC}$ oxidation during normal and malignant germ cell development. PLoS One 2013; 8: e82881, doi: 10.1371/ journal.pone.0082881.

33. Peng B, Hurt EM, Hodge DR, Thomas SB, Farrar WL. DNA hypermethylation and partial gene silencing of human thymine- DNA glycosylase in multiple myeloma cell lines. Epigenetics 2006; 1: 138-145, doi: 10.4161/epi.1.3.2938.

34. Yatsuoka T, Furukawa T, Abe T, Yokoyama T, Sunamura $M$, Kobari $M$, et al. Genomic analysis of the thymine-DNA glycosylase (TDG) gene on 12q22-q24.1 in human pancreatic ductal adenocarcinoma. Int J Pancreatol 1999; 25: 97-102, doi: 10.1385/IJGC:25:2:97.

35. Kim H, Park J, Jung Y, Song SH, Han SW, Oh DY, et al. DNA methyltransferase 3-like affects promoter methylation of thymine DNA glycosylase independently of DNMT1 and DNMT3B in cancer cells. Int J Oncol 2010; 36: 1563-1572.

36. Morita S, Horii T, Kimura M, Ochiya T, Tajima S, Hatada I. miR-29 represses the activities of DNA methyltransferases and DNA demethylases. Int J Mol Sci 2013; 14: 1464714658, doi: 10.3390/ijms140714647.

37. Gallais R, Demay F, Barath P, Finot L, Jurkowska R, Le Guevel R, et al. Deoxyribonucleic acid methyl transferases $3 a$ and $3 b$ associate with the nuclear orphan receptor COUP-TFI during gene activation. Mol Endocrinol 2007; 21 2085-2098, doi: 10.1210/me.2006-0490.

38. Li YQ, Zhou PZ, Zheng XD, Walsh CP, Xu GL. Association of Dnmt3a and thymine DNA glycosylase links DNA methylation with base-excision repair. Nucleic Acids Res 2007; 35: 390-400, doi: 10.1093/nar/gkl1052.

39. Lokk K, Modhukur V, Rajashekar B, Martens K, Magi R, Kolde $\mathrm{R}$, et al. DNA methylome profiling of human tissues identifies global and tissue-specific methylation patterns. Genome Biol 2014; 15: r54, doi: 10.1186/gb-2014-15-4-r54

40. Kamihara J, Rana HQ, Garber JE. Germline TP53 mutations and the changing landscape of Li-Fraumeni syndrome. Hum Mutat 2014; 35: 654-662, doi: 10.1002/humu.22559. 